COVID-19-ассоциированный бивентрикулярный синдром Такоцубо, осложнившийся кардиогенным шоком (клиническое наблюдение)

\author{
Е. М. ЗЕЛТЫНЬ-АБРАМОВ ${ }^{1,2}$, Н. И. БЕЛАВИНА', Н. Н. КЛОЧКОВА, \\ А. М. СВАНАДЗЕ,
}

'Городская клиническая больница № 52, Москва, РФ

Российский национальный исследовательский медицинский университет им. Н. И. Пирогова, Москва, РФ

реди внелегочных проявлений COVID-19 важное место занимает острая миокардиальная дисфункция. Одной из причин ее развития является синдром Такоцубо (CT). Частота встречаемости CT у пациентов с COVID-19 неизвестна. Представлено клиническое наблюдение пожилой пациентки с тяжелым течением COVID-19, получавшей лечение программным гемодиализом. В ходе сеанса терапевтического сопровождавшейся инфарктоподобными изменениями ЭКГ. По данным Эхо-КГ выявлен типичный паттерн бивентрикулярного СТ с выраженным снижением систолической функции обоих желудочков. По результатам экстренной коронароангиографии обструктивного поражения коронарного русла не выявлено. В течение последующей недели лечения отмечались полное восстановление систолической функции обоих желудочков, регресс инфарктоподобных изменений ЭКГ и разрешение кардиогенного шока. Дальнейшее течение заболевания осложнилось развитием сепсиса и полиорганной недостаточности, приведших к летальному исходу. По данным аутопсии коронарогенное и вирусное поражение миокарда были исключены.

Представленный случай демонстрирует развитие жизнеугрожающего варианта СТ у пациентки с COVID-19. B качестве вероятного триггера следует рассматривать процедуру терапевтического плазмообмена.

Ключевые слова: COVID-19, синдром Такоцубо, терапевтический плазмообмен, кардиогенный шок

Для цитирования: Зелтынь-Абрамов Е. М., Белавина Н. И., Клочкова Н. Н., Исхаков Р. Т., Евсюков О. Ю., Артюхина Л. Ю., Сванадзе А. М., Лысенко M. A. COVID-19-ассоциированный бивентрикулярный синдром Такоцубо, осложнившийся кардиогенным шоком (клиническое наблюдение) // Вестник анестезиологии и реаниматологии. - 2022. - T. 19, № 1. - С. 25-31. DOI: 10.21292/2078-5658-2022-19-1-25-31

\title{
COVID-19-Associated Biventricular Takotsubo Syndrome Complicated with Cardiogenic Shock (Clinical Case)
}

\section{E. M. ZELTYN-ABRAMOV', , N. I. BELAVINA', N. N. KLOCHKOVA ${ }^{1,2}$, R. T. ISKHAKOV', O. YU. EVSYUKOV', , L. YU. ARTYUKHINA', A. M. SVANADZE ${ }^{1,2}$, M. A. LYSENKO',2}

${ }^{1}$ City Clinical Hospital no. 52, Moscow, Russia

${ }^{2}$ Pirogov Russian National Research Medical University, Moscow, Russia

Acute myocardial dysfunction (AMD) remains prominent among extrapulmonary manifestations of COVID-19. Takotsubo syndrome (TS) is one of the causes of AMD development. TS incidence in patients with COVID-19 is currently unknown. The report presents a clinical case of an elderly female patient on maintenance hemodialysis with severe COVID-19. During the session of therapeutic plasma exchange (TPE), the patient had an episode of ventricular fibrillation followed by cardiogenic shock development and ECG changes, similar to myocardial infarction. Echocardiographic (Echo) data showed a distinctive pattern of biventricular TS with a significant systolic function decrease in both ventricles. Emergency CT angiography ruled out obstructive lesions of coronary arteries. Full recovery of both ventricles systolic function, regression of ECG changes, and complete resolution of cardiogenic shock were observed within the next week. The subsequent course of the disease was complicated by sepsis and multiple organ failure which determined the lethal outcome. The autopsy findings excluded coronarogenic and viral myocardial damage. The presented case demonstrates a life-threatening TS pattern in patients with severe COVID-19. TPE procedure should be treated as a possible trigger of TS.

Key words: COVID-19, Takotsubo syndrome, therapeutic plasma exchange, cardiogenic shock

For citations: Zeltyn-Abramov E.M., Belavina N.I., Klochkova N.N., Iskhakov R.T., Evsyukov O.Yu., Artyukhina L.Yu., Svanadze A.M., Lysenko M.A. COVID-19-associated biventricular Takotsubo syndrome complicated with cardiogenic shock (clinical case). Messenger of Anesthesiology and Resuscitation, 2022, Vol. 19, no. 1, P. 25-31. (In Russ.) DOI: 10.21292/2078-5658-2022-19-1-25-31

Для корреспондениии:

Зелтынь-Абрамов Евгений Мартынович

E-mail: ezeltyn@mail.ru

Новая коронавирусная инфекция стала серьезным вызовом медицинскому сообществу XXI в. В настоящее время продолжается изучение внелегочных проявлений COVID-19, среди которых прогностически важным является развитие COVID-ассоциированной острой миокардиальной дисфункции $[5,14]$. Острая миокардиальная дисфункция у пациентов с COVID-19 может сопровождаться клиникой острой сердечной недостаточности в диапазоне от отека легких до кардиогенного

\section{Correspondence:}

Evgeny M. Zeltyn-Abramov

Email: ezeltyn@mail.ru

шока, а также манифестацией фатальных аритмий $[5,14]$. Причины острой миокардиальной дисфункции: вирусное поражение миокарда, различные типы инфарктов миокарда вследствие COVID-accoциированной эндотелиальной дисфункции, токсические эффекты медикаментозной терапии и развитие синдрома Такоцубо (СТ) [14]. СТ представляет собой острую миокардиальную дисфункцию, клинически трудно отличимую от острого коронарного синдрома, включая типичный ангинозный приступ 
и повышение концентрации маркеров повреждения миокарда $[9,16]$. Особенностью СТ являются специфические эхокардиографические (Эхо-КГ) паттерны нарушения локальной сократимости миокарда, инфарктоподобные изменения ЭКГ в виде появления глубоких отрицательных зубцов Т и девиации ST-сегмента выше/ниже изолинии $[1,9,11]$. Окончательная диагностика СТ базируется на результатах коронароангиографии, которые подтверждают интактность коронарного русла [9, 16]. Несмотря на то что в большинстве случаев для СТ характерна полная обратимость клинических и инструментальных данных, летальность от него колеблется от 1-2 до 5\%, основные причины неблагоприятных исходов - кардиогенный шок, фатальные желудочковые аритмии и разрыв миокарда левого желудочка [9].

Современные представления о патогенезе СТ базируются на концепции катехоламинового шторма. Избыточный выброс катехоламинов может привести к тяжелому избирательному регионарному оглушению миокарда с развитием острой миокардиальной дисфункции. Ее тяжесть и Эхо-КГ-паттерны при СТ, в отличие от острого коронарного синдрома, определяются не критическим дефицитом коронарного кровотока, а концентрацией $\beta 1$-адренорецепторов, реагирующих на избыток катехоламинов в миокарде конкретного пациента [9, 16]. С точки зрения коронарной анатомии нарушения локальной сократимости при СТ экстерриториальны и не соответствуют бассейну кровоснабжения той или иной коронарной артерии. Триггерами катехоламинового шторма традиционно считаются различные психоэмоциональные или медицинские события (гипоксемия, влияние медикаментов, различные хирургические или диагностические вмешательства и др.). Приблизительно в 30\% случаев окончательная причина развития СТ остается неопределенной $[9,16]$. Частота встречаемости данной патологии у пациентов с COVID-19 до конца не ясна. Целью представленного наблюдения является ознакомление врачей-интенсивистов со случаем СТ, который явился причиной развития тяжелой миокардиальной дисфункции у пациентки с COVID-19.

Клиническое наблюдение. Пациентка 72 лет поступила на 3-и сут заболевания с жалобами на одышку при минимальной физической нагрузке, малопродуктивный кашель, повышение температуры тела до $39,0^{\circ} \mathrm{C}$, частый жидкий стул. Диагноз COVID-19 подтвержден положительным ПЦР-тестом на амбулаторном этапе. В анамнезе: терминальная почечная недостаточность в исходе поликистозной болезни почек, артериальная гипертензия, лечение программным гемодиализом в течение 8 лет. Объективно состояние было расценено как тяжелое: температура тела $38,7^{\circ} \mathrm{C}$, одышка в покое до 20 в мин, $\mathrm{SpO}_{2} 92 \%$ при дыхании атмосферным воздухом, аускультативно - жесткое дыхание, рассеянные трескучие хрипы. Частота сердечных сокращений 90 уд/мин, артериальное давление 130/90 мм рт. ст. На ЭКГ: синусовый ритм, без признаков очаговых изменений, ST изоэлектричен, $\mathrm{QT}_{\mathrm{c}}-404$ мс. Компьютерная томография органов грудной клетки: картина двустороннего поражения легких, типичная для COVID-19 (КT-3).

При поступлении, по данным лабораторных исследований, обращали на себя внимание высокие показатели С-реактивного белка (80 мг/мл, норма 0-6 мг/мл) и лактатдегидрогеназы (400 Ед/л, норма 0-248 Ед/л), лимфопения $\left(0,6 \times 10^{9} /\right.$ л) и тромбоцитопения $\left(45 \times 10^{9} /\right.$ л). С учетом выраженного интоксикационного синдрома и иммуновоспалительного ответа инициировали терапию блокатором интерлейкина-6 (левилимаб 324 мг), начали респираторную поддержку инсуффляцией кислорода через лицевую маску (8-10 л/мин). Несмотря на проводимую терапию, отмечалось усиление дыхательной недостаточности, нарастали концентрации маркеров воспаления (С-реактивный белок 120 мг/мл), прогрессировала тромбоцитопения $\left(30 \times 10^{9} / л\right)$ на фоне снижения уровня гемоглобина (60 г/л) и увеличения показателей лактатдегидрогеназы (600 Ед/л). С целью купирования цитокинового шторма и вторичной тромботической микроангиопатии (COVID-ассоциированного TMA-подобного синдрома) было принято решение о проведении терапевтического плазмообмена. Сеанс терапевтического плазмообмена проводился по стандартной методике в условиях респираторной поддержки и поверхностной медикаментозной седации в отделении реанимации и интенсивной терапии (ОРИТ). В конце процедуры терапевтического плазмообмена у пациентки развился эпизод фибрилляции желудочков с полной остановкой кровообращения. Начаты реанимационные мероприятия, пациентка интубирована и переведена на искусственную вентиляцию легких (ИВЛ), проведена дефибрилляция, со второй попытки восстановлен синусовый ритм. Показатели гемодинамики стабилизированы линейным введением норадреналина. На ЭКГ: синусовый ритм, элевация сегмента ST с формированием глубоких отрицательных зубцов Т, QT $\mathrm{c}-500$ мс (рис. 1). Тропонин I - 170 нг/л (норма до 23 нг/л).

Эхо-КГ-исследование выявило значительное снижение систолической функции обоих желудочков (фракция выброса левого желудочка по Симпсону) - около 20\% (норма > 55\%), FAC RV (Fractional area change) - 25\% (норма > 35\%), циркулярный акинез области верхушки и средних сегментов обоих желудочков, гиперкинез базальных сегментов обоих желудочков (рис. 2). Умеренное расширение левых отделов сердца (индекс конечно-диастолического объема левого желудочка 80 мл/м² (норма до 61 мл/м²), индекс объема левого предсердия 36 мл/м² (норма до 34 мл/м²), размеры правых отделов сердца не увеличены, умеренная легочная гипертензия (систолическое давление в легочной артерии 40 мм рт. ст., норма до 30 мм рт. ст.), умеренная митральная и трикуспидальная регургитация.

Для исключения острого коронарного синдрома как причины эпизода остановки кровообращения 


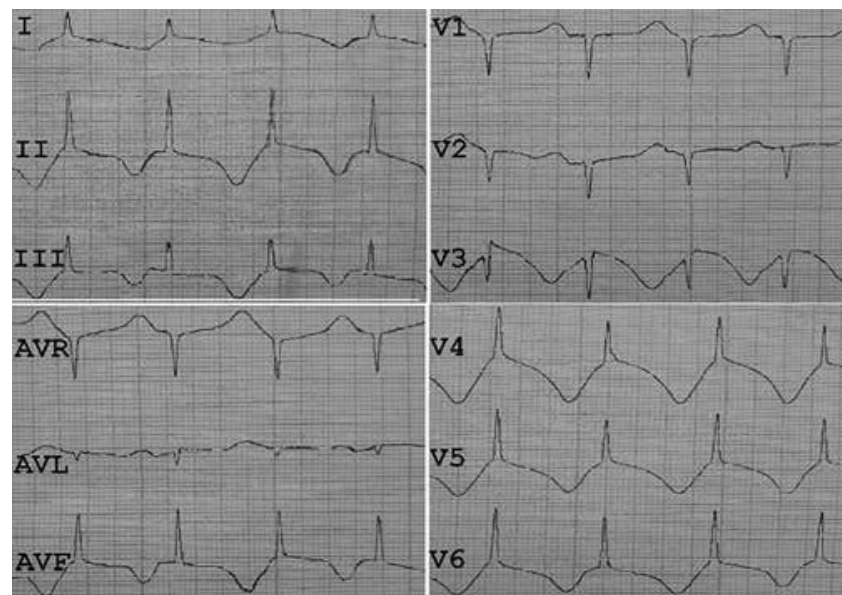

Pис. 1. ЭКГ после эпизода фибрилляции желудочков

Fig. 1. ECG after a ventricular fibrillation episode

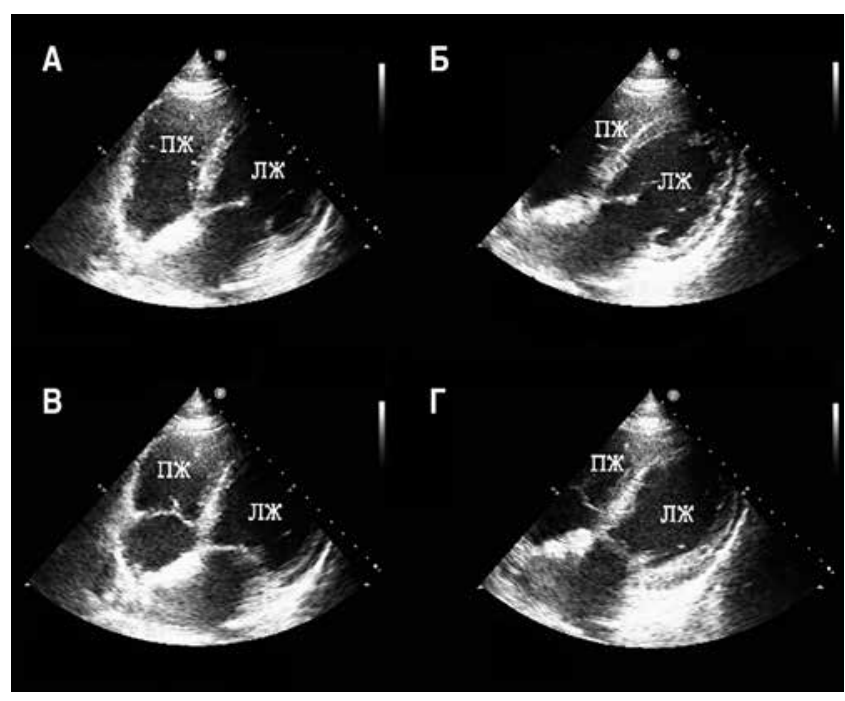

Рис. 2. Эхо-КГ-исследование, субкостальный доступ. А - диастола желудочков, акцент на правый желудочек (ПЖ); Б - диастола желудочков, акцент на левый желудочек (ЛЖ); В - систола желудочков, акчент на ПЖ; Г-систола желудочков, акцент на ЛЖ

Fig. 2. Echo-CG, subcostal access. A - ventricular diastole, emphasis on the right ventricle ( $R V) ; D$ - ventricular diastole, emphasis on the left ventricle ( $L V) ; B$ - ventricular systole, emphasis on the $R V$; $\Gamma$ - ventricular systole, emphasis on the $L V$

и развития кардиогенного шока выполнена экстренная коронароангиография, которая не выявила стенотического поражения коронарных артерий (рис. 3).

Таким образом, с учетом характерного Эхо-КГ-паттерна апикального баллонирования обоих желудочков и данных коронароангиографии установлен диагноз бивентрикулярного СТ, осложнившегося фибрилляцией желудочков с последующим развитием кардиогенного шока. К лечению добавлены левосимендан и небольшие дозы $\beta$-блокаторов. Состояние пациентки оставалось тяжелым, продолжали ИВЛ, параметры гемодинамики под-

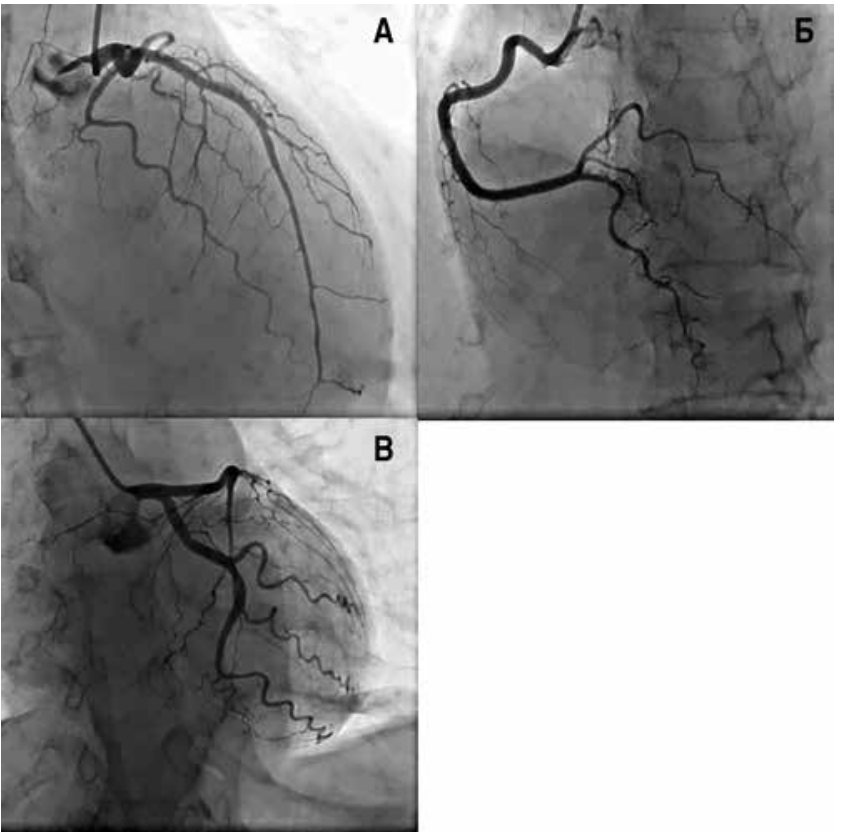

Pис. 3. Коронароангиография. А - передняя межжелудочковая ветвь левой коронарной артерии (ЛКА); Б - правая коронарная артерия; $B$ - огибаюшая ветвь ЛКА

Fig. 3. Coronary angiography. A - anterior interventricular branch of the left coronary artery ( $L C A) ; B$ - right coronary artery; $B$ - circumflex branch of the $L C A$

держивали введением норадреналина. Проведена серия Эхо-КГ-исследований, выявлена последовательная положительная динамика. Через неделю после эпизода фибрилляции желудочков: систолическая функция обоих желудочков восстановилась (фракция выброса левого желудочка 55\%, TAPSE (Tricuspid annular plane systolic excursion) 1,9 cм, норма $>1,7$ см), зоны нарушения локальной сократимости не определялись. Сохранялась умеренная легочная гипертензия (систолическое давление в легочной артерии 45 мм рт. ст.). На ЭКГ отмечался регресс очаговых изменений миокарда - ST изоэлектричен, зубец Т положительный в грудных отведениях, $\mathrm{QT}_{\mathrm{c}}-410$ мс (рис. 4).

Пациентку отлучили от ИВЛ, экстубировали, гемодинамика стабильна, без прессорной поддержки. В дальнейшем течение заболевания осложнилось прогрессированием COVID-ассоциированного поражения легких с присоединением вторичной бактериальной инфекции (в посевах из бронхоальвеолярного лаважа выделены полирезистентные штаммы Acinetobacter baumanii и Klebsiella pneumonia), развитием сепсиса. Непосредственной причиной смерти стало прогрессирование полиорганной недостаточности.

По результатам аутопсии диагноз новой коронавирусной инфекции, осложненной полиорганной недостаточностью и сепсисом, был подтвержден. Данных за наличие гемодинамически значимого стенотического поражения коронарных артерий сердца не получено. Проведенное прицельное изу- 


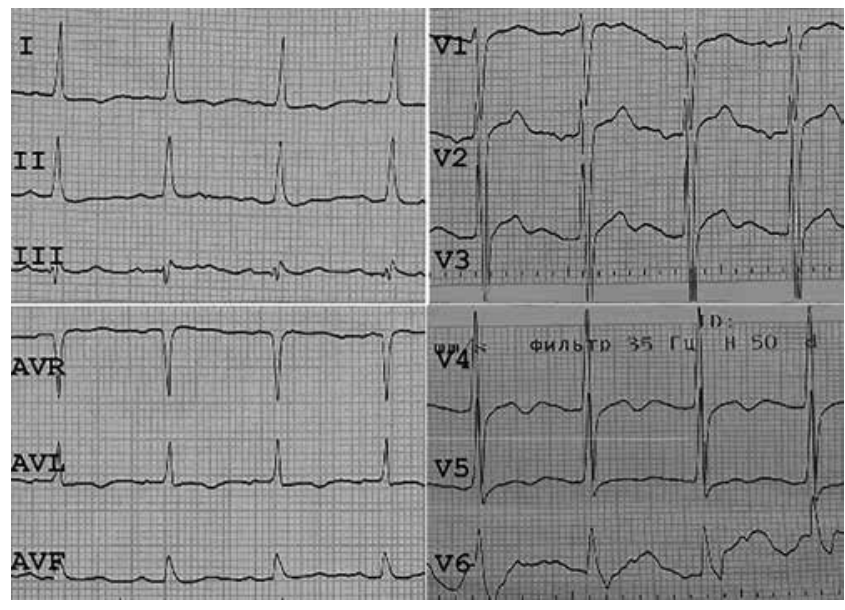

Рис. 4. ЭКГ-динамика через неделю

Fig. 4. ECG changes after one week

чение коронарного русла и гистологическое исследование миокарда позволили исключить воспалительный и коронарогенный генез повреждения кардиомиоцитов. Результаты гистологического исследования миокарда представлены на рис. 5.

\section{Обсуждение}

На фоне высокого уровня психоэмоционального напряжения в обществе, связанного с пандемией новой коронавирусной инфекции, отмечается значительное увеличение частоты встречаемости СТ среди пациентов без верифицированного диагноза COVID-19 [15]. По состоянию на ноябрь 2021 г. опубликовано небольшое количество клинических наблюдений СТ у пациентов с подтвержденным COVID-19 [3, 5-7, 10, 13-15, 17-18, 21, 22]. Примечательно, что данные о частоте встречаемости СТ среди пациентов ОРИТ отсутствуют, несмотря на то что примерно у $22 \%$ реанимационных пациентов имеются клинико-инструментальные признаки острой миокардиальной дисфункции [23]. За период 04.2020 г. - 10.2021 г. среди 1244 пациентов, наблюдавшихся в ОРИТ нефрологического профиля ГБУЗ ГКБ № 52, было проведено 9 экстренных коронароангиографий в связи с подозрением на острый коронарный синдром: в 5 случаях этот диагноз подтвержден, в 4 - коронарное русло оказалось интактным. За этот же период времени диагноз СТ был установлен дважды: у молодой пациентки с фульминантным течением COVID-19 без предшествующей кардиальной патологии в раннем периоде после аллотрансплантации трупной почки [3] и в описываемом клиническом наблюдении. Для пациентов с COVID-19, получающих лечение программным гемодиализом, характерны высокая частота развития фатальных кардиоваскулярных событий и актуализация коморбидной патологии [4], однако в процессе пандемии развитие СТ, по-видимому, встречается не так часто.

Пациенты ОРИТ подвергаются большому количеству медикаментозных и инструментальных

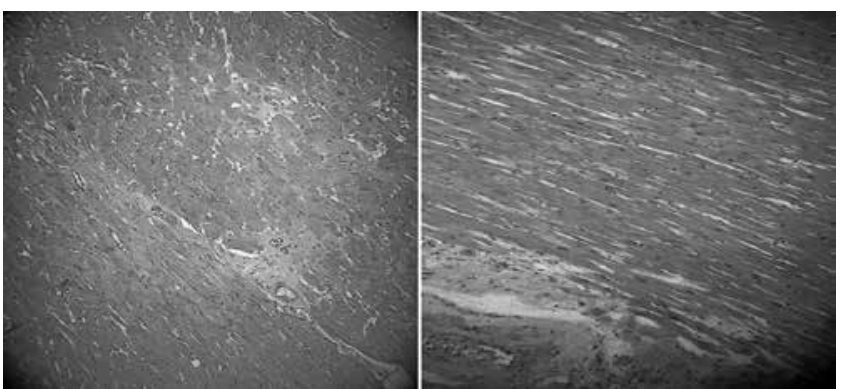

Pис. 5. Гистологическое исследование миокарда. Окраска гематоксилином и эозином, ув. $\times 200$. Кардиомиочиты умеренно увеличены в поперечных размерах, на отдельных участках - извитого вида, имеет место неравномерно выраженная стертость поперечной исчерченности (что может свидетельствовать о контрактурных нарушениях миофибрилл), очаги плазмолиза и фрагментачии мышечных волокон. В цитоплазме многих кардиомиоцитов видны отложения рыхлых эозинофильных депозитов. Ядра с диффузной гиперхромией, округлой и овальной формы, расположены в центральных и периферических отделах ицтоплазмы миоцитов. В строме миокарда на фоне отека и базофилии определяются умеренно дилатированные капилляры с эритростазами, а также мелкие артерии и артериолы с утолщением стенок, частоколообразным расположением эндотелиальных клеток, эритростазами

Fig. 5. Histological examination of the myocardium. Hematoxylin-eosin staining, $\times 200$. Transverse sizes of cardiomyocytes are moderately enlarged, in some areas - tortuous form, there is unevenly expressed erasure of transverse striation (which may indicate contractural disorders of myofibrils), foci of plasmolysis and fragmentation of muscle fibers. The cytoplasm of many cardiomyocytes shows deposits of loose eosinophilic deposits. Nuclei with diffuse hyperchromia, rounded and oval, are located in the central and peripheral parts of myocyte cytoplasm. In myocardial stroma, against edema and basophilia, moderately dilated capillaries with erythrostases are detected, as well as small arteries and arterioles with thickened walls, often spike-like arrangement of endothelial cells, erythrostases.

вмешательств, которые теоретически могут стать триггерами развития СТ даже в условиях медикаментозной седации, исключающей психоэмоциональный фактор. Весьма вероятным триггером развития СТ в данном клиническом наблюдении представляется процедура терапевтического плазмообмена, рекомендуемая рядом исследователей для купирования избыточного системного воспалительного ответа [20]. Окончательно подтвердить или опровергнуть данное предположение не представляется возможным из-за крайне малого количества публикаций на эту тему. В доступной авторам литературе имеется единичное описание развития типичного СТ в ходе процедуры плазмафереза у пациента без COVID-19 [8]. В то же время описан случай полного разрешения клинико-инструментальной картины CT у пациента с COVID-19 после 5 последовательных сеансов терапевтического плазмообмена [12]. По состоянию на ноябрь 2021 г. 
в ОРИТ для пациентов нефрологического профиля ГБУЗ ГКБ № 52 было проведено 1236 процедур терапевтического плазмообмена, и только в описываемом наблюдении развился СТ. Тем не менее стремительное развитие СТ у пациентки со стабильным исходным кардиальным статусом, находившейся в состоянии медикаментозной седации, позволяет с высокой долей вероятности рассматривать данный случай как перипроцедуральное осложнение терапевтического плазмообмена. Бивентрикулярный фенотип является прогностически неблагоприятным вариантом течения СТ независимо от сопутствующих заболеваний и непосредственных триггеров его развития $[1,4,10,11]$. Кроме того, систолическая дисфункция ПЖ - самостоятельный фактор риска неблагоприятного исхода COVID-19 [2], что в сочетании с бивентрикулярным СТ приводит к тяжелым гемодинамическим последствиям. В представленном клиническом наблюдении не проводили прижизненную диагностику COVID-acсоциированного миокардита из-за объективной недоступности ключевых диагностических модальностей (эндомиокардиальная биопсия миокарда и
МРТ сердца с парамагнитным контрастированием) в условиях пандемии. Данное наблюдение демонстрирует исчерпывающий набор диагностических методик, включая гистологическое исследование миокарда post mortem, подтверждающих наличие СТ у пациентки с новой коронавирусной инфекцией.

\section{Заключение}

Впервые представлен случай бивентрикулярного СТ, осложнившегося развитием фибрилляции желудочков и кардиогенного шока у пациентки c COVID-19. В реальной клинической практике необходимо: 1) включать СТ в дифференциально-диагностический поиск причин острой миокардиальной дисфункции при COVID-19; 2) Эхо-КГ рассматривать в качестве основной диагностической модальности в режиме «bedside» для выявления СТ; 3) у пожилых коморбидных пациентов проводить коронароангиографию для окончательного исключения острого коронарного синдрома как причины острой миокардиальной дисфункции.

Конфликт интересов. Авторы заявляют об отсутствии у них конфликта интересов.

Conflict of Interests. The authors state that they have no conflict of interests.

\section{ЛИТЕРАТУРА}

1. Алехин М. Н. Синдром Такоцубо: значение эхокардиографии // Кардиология. - 2017. - Т. 57, № 7. - С. 80-89. https://doi.org/10.18087/cardio.2017.7.10009.

2. Голухова Е. 3., Сливнева И. В., Рыбка М. М. и др. Систолическая дисфункция правого желудочка как предиктор неблагоприятного исхода у пациентов с COVID-19 // Кардиология. - 2020. - T. 60, № 11. - С. 16-29. https://doi.org/10.18087/cardio.2020.11.n1303.

3. Зелтынь-Абрамов Е. М., Белавина Н. И., Фролова Н. Ф. и др. Стресс-индуцированная кардиомиопатия у пациентки с фульминантным течением COVID-19 в ранние сроки после аллотрансплантации почки (наблюдение из практики и краткий обзор литературы) // Клиническая нефрология. - 2020. - № 2. - С. 10-15. doi: https://dx.doi. org/10.18565/nephrology.2020.2.10-15

4. Зелтынь-Абрамов Е. М., Белавина Н. И., Фролова Н. Ф. и др. Факторы риска неблагоприятного прогноза у пациентов на программном гемодиализе с COVID-19. Акцент на сердечно-сосудистую коморбидность. Опыт одного центра // Нефрология и диализ. - 2020. - Т. 22, № 3. - С. 9-56. https://doi.org/10.28996/2618-9801-2020-Special_Issue-9-20.

5. Козлов И. А., Тюрин И. Н. Сердечно-сосудистые осложнения COVID-19// Вестник анестезиологии и реаниматологии. - 2020. - Т. 17, № 4. - С. 14-22. https://doi.org/10.21292/2078-5658-2020-17-4-14-22.

6. Устинова Е. С., Яковлев А. А., Марзаева Е. В. и др. Сложности дифференциальной диагностики синдрома Такоцубо и острого инфаркта миокарда на фоне новой коронавирусной инфекции COVID-19 // Кардиология: новости, мнения, обучение. - 2021. - Т. 9, № 1. - С. 59-64. doi: https://doi. org/10.33029/2309-1908-2021-9-1-59-64.

7. Almas T., Khedro T., Haadi A. et al. COVID-19-induced Takotsubo cardiomyopathy: Venturing beyond the obvious // Ann. Med. Surg. (Lond). 2021. - Vol. 65. - P. 102291. doi: 10.1016/j.amsu.2021.102291. PMID: 33981423; PMCID: PMC8082200.

8. Arai M., Ukigai H., Miyata H. A case of transient left ventricular ballooning ("Takotsubo"-shaped cardiomyopathy) developed during plasmapheresis for treatment of myasthenic crisis] // Rinsho Shinkeigaku. - 2004. - Vol. 44, № 3 P. 207-210. Japanese. PMID: 15233276.

9. Cammann V. L., Würdinger M., Ghadri J. R. et al. Takotsubo syndrome: uncovering myths and misconceptions // Curr. Atheroscler. Rep. -2021. - Vol. 3, № 9. - P. 53. Published 2021 Jul 16. doi:10.1007/s11883-021-00946-z.

\section{REFERENCES}

1. Alekhin M.N. Takotsubo syndrome: the meaning of echocardiography. Kardiologia, 2017, vol. 57, no. 7, pp. 80-89. (In Russ.) https://doi. org/10.18087/cardio.2017.7.10009.

2. Golukhova E.Z., Slivneva I.V., Rybka M.M. et al. Right ventricular systolic dysfunction as a predictor of adverse outcome in patients with COVID-19. Kardiologia, 2020, vol. 60, no. 11, pp. 16-29. (In Russ.) https://doi. org/10.18087/cardio.2020.11.n1303.

3. Zeltyn-Abramov E.M., Belavina N.I., Frolova N.F. et al. Stress-induced cardiomyopathy in a patient with a fulminant course of COVID-19 early after a kidney allotransplantation. Klinicheskaya Nefrologiya, 2020, no. 2, pp. 10-15. (In Russ.) doi: https://dx.doi.org/10.18565/nephrology.2020.2.10-15.

4. Zeltyn-Abramov E.M., Belavina N.I., Frolova N.F. et al. Risk factors of adverse outcome of COVID-19 and experience of Tocilizumab administration in patients on maintenance hemodialysis due to diabetic kidney disease. Nefrologiya i Dializ, 2020, vol. 22, no. 3, pp. 9-56. (In Russ.) https://doi.org/10 .28996/2618-9801-2020-Special_Issue-9-20.

5. Kozlov I.A., Tyurin I.N. Cardiovascular complications of COVID-19 Messenger of Anesthesiology and Resuscitation, 2020, vol. 17, no. 4, pp. 14-22. (In Russ.) https://doi.org/10.21292/2078-5658-2020-17-4-14-22.

6. Ustinova E.S., Yakovlev A.A., Marzaeva E.V. et al. Differential diagnostics of Takotsubo syndrome and acute myocardial infarction against the background of a new COVID-19 coronavirus infection. Kardiologiya Novosti, Mneniya, Obuchenie, 2021, vol. 9, no. 1, pp. 59-64. (In Russ.) doi: https://doi.org/10.33 029/2309-1908-2021-9-1-59-64.

7. Almas T., Khedro T., Haadi A. et al. COVID-19-induced Takotsubo cardiomyopathy: Venturing beyond the obvious. Ann. Med. Surg. (Lond)., 2021, vol. 65, pp. 102291. doi: 10.1016/j.amsu.2021.102291. PMID: 33981423; PMCID: PMC8082200.

8. Arai M., Ukigai H., Miyata H. A case of transient left ventricular ballooning ("Takotsubo"-shaped cardiomyopathy) developed during plasmapheresis for treatment of myasthenic crisis]. Rinsho Shinkeigaku, 2004, vol. 44, no. 3, pp. 207-210. Japanese. PMID: 15233276.

9. Cammann V.L., Würdinger M., Ghadri J.R. et al. Takotsubo syndrome: uncovering myths and misconceptions. Curr. Atheroscler. Rep., 2021, vol. 3, no. 9, pp. 53. Published 2021 Jul 16. doi:10.1007/s11883-021-00946-z. 
10. Citro R., Bossone E., Parodi G. et al. Independent impact of RV involvement on in-hospital outcome of patients with Takotsubo syndrome // JACC Cardiovasc. Imaging. - 2016. - Vol. 9, № 7. - P. 894-895. doi: 10.1016/j.jcmg.2015.06.005

11. Citro R., Lyon A. R., Meimoun P. et al. Standard and advanced echocardiography in Takotsubo (stress) cardiomyopathy: clinical and prognostic implications // J. Am. Soc. Echocardiogr. - 2015. - Vol. 28, № 1. - P. 57-74. doi:10.1016/j. echo.2014.08.020.

12. Faqihi F., Alharthy A., Alshaya R. et al. Reverse takotsubo cardiomyopathy in fulminant COVID-19 associated with cytokine release syndrome and resolution following therapeutic plasma exchange: a case-report // BMC Cardiovasc Disord. - 2020. - Vol. 20, № 1 - P. 389. doi: 10.1186/s12872-020-01665-0. PMID: 32842957; PMCID: PMC7447602.

13. Fujisaki T., Kassim F., Kassim G. et al. Biventricular takotsubo syndrome with COVID-19 in an Asian male // J. Cardiol. Cases. - 2021. - Vol. 24, № 1 - P. 6-9. doi: 10.1016/j.jccase.2020.11.017. Epub 2020 Nov 26. PMID: 33262862; PMCID: PMC7690273.

14. Gupta A., Madhavan M. V., Sehgal K. et al. Extrapulmonary manifestations of COVID-19 // Nat. Med. - 2020. - Vol. 26, № 7. - P. 1017-1032. https://doi. org/10.1038/s41591-020-0968-3.

15. Jabri A., Kalra A., Kumar A. et al. Incidence of Stress Cardiomyopathy During the Coronavirus Disease 2019 Pandemic // JAMA Netw Open. - 2020. - Vol. 3, № 7. P. e2014780. Published 2020 Jul 1. doi:10.1001/jamanetworkopen.2020.14780.

16. Kato K., Lyon A. R., Ghadri J. R. et al. Takotsubo syndrome: aetiology, presentation and treatment // Heart. - 2017. - Vol. 103, № 18 - P. 1461-1469. doi:10.1136/heartjnl-2016-309783.

17. Okura H. Update of Takotsubo syndrome in the era of COVID-19// J. Cardiol. 2021. - Vol. 77, № 4 - P. 361-369. doi:10.1016/j.jjcc.2020.10.004

18. Shojaei F., Habibi Z., Goudarzi S. et al. COVID-19: A double threat to takotsubo cardiomyopathy and spontaneous coronary artery dissection? // Med. Hypotheses. - 2021. - Vol. 146. - P. 110410. doi:10.1016/j.mehy.2020.110410.

19. Singh S., Desai R., Gandhi Z. et al. Takotsubo syndrome in patients with COVID-19: a systematic review of published cases [published online ahead of print, 2020 Oct 6] // SN Compr. Clin. Med. - 2020. - P. 1-7. doi:10.1007/s42399-020-00557-w.

20. Tabibi S., Tabibi T., Conic R. R. Z. et al. Therapeutic plasma exchange: a potentia management strategy for critically ill COVID-19 patients // J. Intens. Care Med. - 2020. - Vol. 35, № 9. - P. 827-835. doi:10.1177/0885066620940259.

21. Torabi A. J., Villegas-Galaviz J., Guglin M. et al. Cardiogenic shock following cardiac tamponade and Takotsubo in COVID-19 // Future Cardiol. - 2021. Vol. 17, № 4 - P. 631-635. doi: 10.2217/fca-2020-0115. Epub 2020 Oct 20. PMID: 33078963; PMCID: PMC7574646.

22. van Osch D., Asselbergs F. W., Teske A. J. Takotsubo cardiomyopathy in COVID-19: a case report. Haemodynamic and therapeutic considerations // Eur. Heart J. Case Rep. - 2020. - Vol. 27, № 4 (FI1) - P. 1-6. doi: 10.1093/ehjcr/ytaa271. PMID: 33437922; PMCID: PMC7528942.

23. Wang D., Hu B., Hu C. et al. Clinical characteristics of 138 hospitalized patient with 2019 Novel Coronavirus-Infected Pneumonia in Wuhan, China [published correction appears in JAMA 2021 Mar 16;325(11):1113] // JAMA. - 2020. Vol. 323, № 11. - P. 1061-1069. doi:10.1001/jama.2020.1585.

\section{ИНФОРМАЦИЯ ОБ АВТОРАХ:}

ГБУЗ «Городская клиническая больница № 52 ДЗМ», 123182, Москва, ул. Пехотная, д. 3/2.

тел./факс: +7 (499) 196-10-12.

\section{Зелтынь-Абрамов Евгений Мартынович}

доктор медицинских наук, врач-кардиолог.

E-mail: ezeltyn@mail.ru

\section{Белавина Наталья Ивановна}

кандидат медицинских наук, врач отделения

ультразвуковой и функциональной диагностики.

E-mail:natbelavina@mail.ru
10. Citro R., Bossone E., Parodi G. et al. Independent impact of RV involvement on in-hospital outcome of patients with Takotsubo syndrome. JACC Cardiovasc. Imaging, 2016, vol. 9, no. 7, pp. 894-895. doi: 10.1016/j.jcmg.2015.06.005.

11. Citro R., Lyon A.R., Meimoun P. et al. Standard and advanced echocardiography in Takotsubo (stress) cardiomyopathy: clinical and prognostic implications. J. Am. Soc. Echocardiogr., 2015, vol. 28, no. 1, pp. 57-74. doi:10.1016/ j.echo.2014.08.020.

12. Faqihi F., Alharthy A., Alshaya R. et al. Reverse takotsubo cardiomyopathy in fulminant COVID-19 associated with cytokine release syndrome and resolution following therapeutic plasma exchange: a case-report. BMC Cardiovasc. Disord., 2020, vol. 20, no. 1, pp. 389. doi: 10.1186/s12872-020-01665-0. PMID: 32842957; PMCID: PMC7447602.

13. Fujisaki T., Kassim F., Kassim G. et al. Biventricular takotsubo syndrome with COVID-19 in an Asian male. J. Cardiol. Cases, 2021, vol. 24, no. 1, pp. 6-9. doi: 10.1016/j.jccase.2020.11.017. Epub 2020 Nov 26. PMID: 33262862; PMCID: PMC7690273.

14. Gupta A., Madhavan M.V., Sehgal K. et al. Extrapulmonary manifestations of COVID-19. Nat. Med., 2020, vol. 26, no. 7, pp. 1017-1032. https://doi. org/10.1038/s41591-020-0968-3.

15. Jabri A., Kalra A., Kumar A. et al. Incidence of Stress Cardiomyopathy During the Coronavirus Disease 2019 Pandemic. JAMA Netw Open, 2020, vol. 3, no. 7, pp. e2014780. Published 2020 Jul 1. doi:10.1001/jamanetworkopen.2020.14780.

16. Kato K., Lyon A.R., Ghadri J.R. et al. Takotsubo syndrome: aetiology, presentation and treatment. Heart, 2017, vol. 103, no. 18, pp. 1461-1469. doi:10.1136/heartjnl-2016-309783.

17. Okura H. Update of Takotsubo syndrome in the era of COVID-19. J. Cardiol., 2021, vol. 77, no. 4, pp. 361-369. doi:10.1016/j.jjcc.2020.10.004.

18. Shojaei F., Habibi Z., Goudarzi S. et al. COVID-19: A double threat to takotsubo cardiomyopathy and spontaneous coronary artery dissection? Med. Hypotheses, 2021, vol. 146, pp. 110410. doi:10.1016/j.mehy.2020.110410.

19. Singh S, Desai R., Gandhi Z et al. Takotsubo syndrome in patients with COVID-19: a systematic review of published cases [published online ahead of print, 2020 Oct 6]. SN Compr. Clin. Med., 2020, pp. 1-7. doi:10.1007/s42399-020-00557-w.

20. Tabibi S., Tabibi T., Conic R.R.Z. et al. Therapeutic plasma exchange: a potentia management strategy for critically ill COVID-19 patients. J. Intens. Care Med., 2020, vol. 35, no. 9, pp. 827-835. doi:10.1177/0885066620940259.

21. Torabi A.J., Villegas-Galaviz J., Guglin M. et al. Cardiogenic shock following cardiac tamponade and Takotsubo in COVID-19. Future Cardiol., 2021, vol. 17, no. 4, pp. 631-635. doi: 10.2217/fca-2020-0115. Epub 2020 Oct 20. PMID: 33078963; PMCID: PMC7574646.

22. van Osch D., Asselbergs F.W., Teske A.J. Takotsubo cardiomyopathy in COVID-19: a case report. Haemodynamic and therapeutic considerations. Eur. Heart J. Case Rep., 2020, vol. 27, no. 4(FI1), pp. 1-6. doi: 10.1093/ehjcr/ytaa271. PMID: 33437922; PMCID: PMC7528942.

23. Wang D. Hu B, Hu C et al Clinical characteristics of 138 hospitalized patient with 2019 Novel Coronavirus-Infected Pneumonia in Wuhan, China [published correction appears in JAMA 2021 Mar 16;325(11):1113]. JAMA, 2020, vol. 323, no. 11, pp. 1061-1069. doi:10.1001/jama.2020.1585.

\section{INFORMATION ABOUT AUTHORS:}

City Clinical Hospital no. 52, Moscow Health Department, 3/2, Pekhotnaya St., Moscow, 123182.

Phone/Fax: + 7 (499) 196-10-12.

\section{Evgeny M. Zeltyn-Abramov}

Doctor of Medical Sciences, Cardiologist.

Email:ezeltyn@mail.ru

\section{Natalya I. Belavina}

Candidate of Medical Sciences, Physician of Ultrasound and Functional Diagnostics Department.

Email:natbelavina@mail.ru 


\section{Клочкова Наталия Николаевна}

врач отделения ультразвуковой и функциональной диагностики.

E-mail:natalidoc@yandex.ru

\section{Исхаков Рустам Тимурович}

заведующий отделением реанимации и интенсивной терапии больных нефрологического профиля.

E-mail: stamius@yandex.ru

\section{Евсюков Олег Юрвевич}

кандидат медииинских наук

врач патологоанатомического отделения.

E-mail:evs-ol@mail.ru

\section{Артюхина Людмила Юрвевна}

кандидат медицинских наук, заведующая нефрологическим отделением № 1 (патологии трансплантированной почки)

E-mail: arlyu-1404@yandex.ru

\section{Сванадзе Анна Мурадовна}

кандидат медицинских наук, врач отделения ультразвуковой и функциональной диагностики.

E-mail:asvanadze@mail.ru

\section{Льсенко Марьяна Анатольевна}

доктор медищинских наук, главный врач.

E-mail:gkb52@zdrav.mos.ru
Natalia N. Klochkova

Physician of Ultrasound and Functional Diagnostics

Department.

Email:natalidoc@yandex.ru

\section{Rustam T. Iskhakov}

Head of Nephrology Intensive

Care Unit.

Email:stamius@yandex.ru

Oleg Yu. Evsyukov

Candidate of Medical Sciences,

Physician of Pathology Department.

Email:evs-ol@mail.ru

\section{Lyudmila Yu. Artyukhina}

Candidate of Medical Sciences, Head of Nephrology Department no. 1 (Transplanted Kidney Pathology).

Email: arlyu-1404@yandex.ru

\section{Anna M. Svanadze}

Candidate of Medical Sciences, Physician of Ultrasound and Functional Diagnostics Department.

Email: asvanadze@mail.ru

\section{Maryana A. Lysenko}

Doctor of Medical Sciences, Head Physician.

Email:gkb52@zdrav.mos.ru 